\title{
A Conversation Between Nora Volkow and Johannes Czernin
}

\author{
Nora Volkow $^{1}$ and Johannes Czernin ${ }^{2}$ \\ ${ }^{I}$ National Institute on Drug Abuse at the National Institutes of Health, Bethesda, Maryland; and ${ }^{2}$ David Geffen School of Medicine at \\ UCLA, Los Angeles, California
}

$\mathbf{N}$ breaking discoveries of measurable and persistent neurochemical and functional deficits in the brain of the human drug abuser and for her sustained creative efforts to bring scientific rigor and clarity to our understanding of the molecular mechanisms underlying human addiction and other brain diseases. Her research has been transformative in shifting the paradigm of drug addiction from that of a behavioral choice to a brain disease. She pioneered the use of brain imaging to investigate the (1) toxic effects of drugs; (2) molecular mechanisms underlying drugs' rewarding effects; and (3) neurochemical and functional changes that occur with addiction directly in the human brain.

Over the past 30 years, Nora Volkow has published more than 770 peer-reviewed articles, more than 100 book chapters and nonpeer-reviewed manuscripts and edited four books on neuroimaging and addiction and co-edited an Encyclopedia of Neuroscience (co-edited with Donald Pfaff). Over the past 16 years she has been Director of National Institute on Drug Abuse and her leadership has advanced the recognition of addiction as a chronic disease of the brain that should be treated and not criminalized.

She has received multiple awards including membership in the National Academy of Medicine in 2000 and the International Prize for Science from the French Institute of Health and Medical Research in 2009. She was named one of Time magazine's "Top 100 People Who Shape Our World," "One of the 20 People to Watch" by Newsweek magazine, and "Innovator of the Year" by U.S. News \& World Report.

Dr. Czernin: Dr. Volkow, you have a background in psychiatry, nuclear medicine, molecular imaging, and medicine. You held leadership positions within the U.S. Department of Energy, the State University of New York Stony Brook, and you are heading the National Institute on Drug Abuse (NIDA) at the National Institutes of Health (NIH). Because of your diverse knowledge and background, you have unique insights into various manifestations of addiction and the ways in which imaging could help to provide insights and solutions.

I would like to discuss the current opioid crisis and potential lines of attack. Epidemiological numbers are indeed shocking. Opioids alone account for close to 50,000 annual deaths in the United States each year. To put this in perspective, this is 20,000 casualties more than from prostate cancer and 10,000 more than from breast cancer.

You talk often about misconceptions about addiction and about preventive strategies and novel therapeutic approaches. What can be done to address this crisis in terms of better understanding and treating the disease?

COPYRIGHT @ 2019 by the Society of Nuclear Medicine and Molecular Imaging.
Dr. Volkow: There is an urgency to deploy and use scientific knowledge to prevent people from becoming addicted and dying from opioids. One of the most powerful tools I have used consistently for exploring the phenomenon of addiction is brain imaging and, particularly, molecular imaging. These techniques enable us to investigate how opioids interact with the brain and how they influence multiple signaling circuits. Brain imaging has shown that

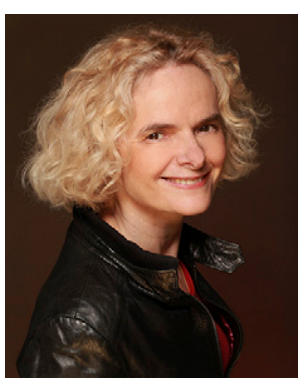

Nora Volkow, MD addiction is a disease of the brain-that it modifies certain areas of the brain in ways that help explain why some people are unable to stop taking a drug despite knowing the high price they are likely to pay. In many instances, death is the ultimate price. People are dying because they cannot control the overwhelming urge to consume the drug, even though they are aware of how dangerous heroin (frequently laced with fentanyl) can be. Furthermore, even prescription opioids can be tainted with fentanyl when procured on the black market. Yet, even when addicted individuals are aware of the potential for catastrophic or fatal outcomes they cannot NOT do it. So the question is: What has changed in the brain of a person who faces the threat of death, of losing custody of his or her children, of losing a job and family, or of being rejected by society, all of which are very big prices to pay, just to get high? Brain imaging has been the technical breakthrough that has allowed us to understand the brain pathophysiology of human addiction in unprecedented detail. Although animal studies have been extraordinarily valuable in elucidating multiple aspects of the neuroscience of drugs and addiction, ultimately the complexity of the human brain can be addressed only by studying humans, which imaging has made possible.

Dr. Czernin: You have often addressed misconceptions about addiction. You stated that there is no addict who wants to be an addict. How does the disease start? Is it a genetic predisposition to become addicted, or is it more an environmental problem?

Dr. Volkow: There are 2 very different questions we need to investigate. The first is: What are the factors that lead a person to start experimenting with drugs? The second is: What are the factors that lead a regular user to become addicted? The fact is that many people experiment with drugs yet never become addicted. There is clear evidence that genetic factors play a role both in experimentation with drugs and also in the transition to addiction. But there is also evidence that environmental factors are extraordinarily important in determining and modulating the extent to which any given person will experiment with drugs and, in doing so, risk becoming addicted. Regardless of net genetic vulnerability, if a person is in an environment in which drugs are widely available or has been exposed to neglect or abuse, other social stressors, or minimal support during childhood or adolescence, the risk of that person using 
drugs as a way to escape such adverse circumstances will increase. Genetics will also influence the vulnerability or resilience of an individual to adverse environmental exposures. Therefore, environmental and genetic factors, as well as their interactions, all modulate overall vulnerability to addiction. Genetic factors also help shape personality or temperament and, through them, can influence the propensity to experiment with drugs. A good example of this effect in action is the personality trait referred to as "novelty seeking." When people experiment with drugs, depending on the drug, they have a roughly $10 \%$ to $20 \%$ chance of becoming addicted. The younger you start the greater is the addiction risk. One of the ways in which genetics influence vulnerability to addiction is by modulating how quickly a person becomes conditioned to the effects of a drug, which then triggers craving on further exposure to the drug or to stimuli associated with it. Apart from genetics, very stressful environments, with a paucity of alternative reinforcers, also increase the vulnerability to seek out drugs as a means to activate the reward circuitry, which, with repeated exposure, can ultimately lead to addiction.

In summary, 3 key factors modulate the transition from experimentation to addiction: genetics, the social environment, and the availability of drugs, all of which are relevant to the current crisis. Policies can play a role in either facilitating or hindering drug addiction by reducing exposure to these drugs. This explains why the use of the legal drugs tobacco and alcohol has been linked to the highest rates of morbidity and mortality from drugs around the world. The importance of drug availability becomes clear in the
Dr. Volkow: Experimental studies show that if you expose animals to THC you modify their sensitivity to other types of drugs like morphine, opioids, nicotine, or cocaine. Human epidemiological studies reveal that adolescents who take marijuana run a greater risk of moving on to other drugs when they are older and have easier access. However, the transition from cannabis to the other drugs may simply reflect patterns of accessibility (as opposed to brain changes triggered by cannabis) that make consumers more vulnerable to the rewarding effects of other drugs.

However, the animal data provide evidence of the possibility that marijuana may prime the brain for the rewarding effects of other drugs, even though current evidence does not allow us to establish causality. Unfortunately, sometimes there is a tendency to exaggerate findings in either direction (for cannabis, these directions are either very addictive and harmful or nonaddictive and innocuous). We do know that cannabis is less addictive than other drugs like nicotine, heroin, or cocaine, but there is unequivocal evidence that it is addictive. Cannabis also interferes with cognitive processes in the brain and the capacity to learn, memorize, and sustain long-term attention. When teenagers consume cannabis, they may end up with poor educational outcomes and increased risk of dropping out of school. To understand how cannabis and other drugs influence the development of the human brain, researchers are taking advantage of imaging tools. Using MRI (because PET use in children is limited by radiation exposure), studies are evaluating whether the use of cannabis during

\section{"Although medications (methadone, buprenorphine, and naltrexone) significantly improve clinical outcomes and can prevent overdoses in OUD, major challenges remain regarding the use of these medications, patient compliance, and relapse rates. Thus, there is a clear need to develop extended formulations to facilitate compliance as well as new treatment strategies to enhance success with recovery."}

example of nicotine addiction, which was nonexistent in the 19th century when there was no access to cigarettes. Such observations underscore the importance of socioeconomic and cultural factors in addiction. However, this is not different from diabetes and obesity, where genetics, socioeconomic, and cultural factors play equally important roles.

Dr. Czernin: You just mentioned that $10 \%$ to $20 \%$ of people who experiment may run into a real addiction problem. How do you view the legalization of cannabis products for recreational use? Is this a risk factor for subsequent drug addiction?

Dr. Volkow: The data clearly show that legal drugs increase the number of people who are exposed to them, as is the case for alcohol and nicotine. The more people who are exposed to a drug the greater the number who will become addicted. We know that $9 \%$ percent of people exposed to marijuana become addicted. If you have only 300 people who are exposed to it, then $9 \%$ is a small number $(\sim 27)$; however, if 3 million people are exposed to it, the total number of people who will become addicted expands to around 270,000 . As a country, we are already starting to see an increase in the number of people consuming cannabis along with significant increases in admissions to emergency departments associated with its consumption. This was expected, because once you get more people exposed to cannabis, then greater numbers will suffer the adverse effects linked to its consumption.

Dr. Czernin: Do you think that tetrahydrocannabinol (THC) is a gateway to the use of more addictive drugs? Are there data to show that? adolescence interferes with brain development. This is crucial, because the endogenous cannabinoid system in our brains regulates multiple processes relevant to brain development and its proper function. For example, the process by which neurons connect with one another is modulated by endogenous cannabinoids, which could be disrupted when artificially stimulated by the consumption of cannabis. Thus, we are particularly concerned about the deleterious effects that cannabis may have on the brains of not only children and adolescents but also the unborn child and neonates. In fact, we are currently funding prospective studies to determine whether early exposure to cannabis could affect the function of the brain's reward system in ways that would make an individual more vulnerable to addiction later in life. Findings of molecular imaging studies of the dopaminergic system using PET have shown that, in addicted individuals and those at risk for addiction, the dopamine reward system is hypofunctional, which on the one hand makes it less sensitive to natural rewards but on the other puts it at higher risk of seeking stronger reinforcers that can activate it, such as is the case for drugs. Imagine what it must be like if everyday actions, like reading books or going to the movies or meeting friends, no longer activate the reward system and motivate you and only the consumption of drugs excites you. Our brain is hardwired to seek out things that motivate us, like seeking and eating food when we are hungry. Reward and motivation is what engages us into action, and, if early cannabis exposure renders the motivational system hypofunctional, this could increase vulnerability to other addictions later on. 
Dr. Czernin: Do we know when and how the dopamine signaling imbalance starts?

Dr. Volkow: There are genetic differences in reward system reactivity. This is not different from genetic factors that determine how fast we run. A certain level can be achieved by training, but inherent capabilities also contribute. We also know that very repressive, isolated, and abusive environments degrade the function of the dopaminergic reward system. That is likely one of the mechanisms that make such environments increase the risk of addiction.

Dr. Czernin: What are potential preventive strategies? They have not been very successful to date. Data coming from NIDA suggest that prescription drugs play a highly significant role in the opioid crisis. Can this be one of the major lines of attack against the problem?

Dr. Volkow: This is an extremely important line of attack. We need to address the widespread availability of prescription opioids. We need to reduce the likelihood that patients who do not really need these medications get them and then misuse them or become addicted. But this is not sufficient. This epidemic started with prescription opioids, but now we are seeing more and more people moving directly into heroin. That is why we also need to develop prevention programs that will protect people from heroin consumption. We should use epidemiological data as our guide: the rates of heroin abuse among teenagers are very low, but we see a very sharp uptick in consumption of opioids during the transition from 18 to $24 \mathrm{y}$ of age. Thus, NIDA is targeting the transition between adolescence to young adulthood as an area for expanding research in development of prevention strategies for opioid use disorders.

Dr. Czernin: You promoted in public statements the need for developing nonaddictive pain medications. What is the status of these efforts and developments? Could imaging help in designing these drugs and then monitoring their effectiveness in terms of pain management? Is there a major push in the pharmaceutical industry to switch from opioids to nonaddictive pain drugs?

Dr. Volkow: Pharma has been mostly disengaged from efforts to develop new, nonaddictive analgesic drugs because these efforts have not been very successful in getting new pain medications into the clinic. The process is very expensive, so, at the NIH, we try to provide incentives through public/private partnerships. We can, for example, "de-risk" compounds to make them more appealing for industry to then take them over and do the work necessary to bring them into the clinic (i.e., conduct 2 or 3 clinical trials).

Dr. Czernin: One can also potentially look at target inhibition and dose optimization. This may be another really important imaging contribution.

Dr. Volkow: Imaging allows us to directly measure the engagement of the drug to its molecular target in the brain and also to monitor how it affects downstream systems, which is extraordinarily valuable for drug development. But imaging is also valuable in that it may help us better characterize patients with pain or with addiction. For example, lower back pain, which is the main cause of pain and disability in the country, can be produced by trauma or neuroinflammation or be associated with altered nerve signaling. Imaging could help determine the underlying etiology of pain, as exemplified by a PET study from the Massachusetts General Hospital that measured translocator protein (TSPO) expression as a marker of neuroinflammation in patients with low back pain and documented a significant upregulation of TSPO in the thalamus. It so happens that TSPO agonists have analgesic effects in animal models, and this biomarker perhaps may serve to predict which patients might be most responsive to such drugs. Thus, a priority for
$\mathrm{NIH}$ is to help researchers develop imaging biomarkers that can help drug development for analgesics and addiction.

Dr. Czernin: You often talk about drug addiction in the context of the dopaminergic reward system, and you discuss obesity in the same context. Nobody wants to be obese. Obesity is, of course, a major health issue with metabolic syndromes, diabetes, hyperlipidemia, and increased risk for cardiovascular events. How can you engage society, researchers, health policy makers, and industry to combat this epidemic?

Dr. Volkow: Imaging has helped us understand how certain foods, particularly those with high sugar or fat content, can disrupt the function and biochemistry of the brain. Can we use this information to actually develop policies and to also educate people about the adverse consequences of certain diets? This is no different from changing the culture of stigmatization of addiction. It puts obesity under the broader category of preventable and treatable diseases. The brains of obese patients are directly modified by exposure to high-caloric foods. A very important target of these diets is the dopaminergic system, which, in turn affects locomotor activity. That is why obese patients are much less motivated to be physically active. Obesity downregulates the responsiveness of the dopaminergic system, just like in addiction. Some obese individuals, with deficits in this system, may overeat in an attempt to compensate for that deficit.

Dr. Czernin: The food industry plays an important role. Changing the diet of children seems to be difficult. We have a culture of bad food. Fast food is much less expensive than what some people call slow food or healthy food. How can this culture be changed?

Dr. Volkow: Policies can do much more for public health than anything else. Taxes would be one way to approach this. This has worked very well for alcohol and tobacco. So why not increase taxes on foods that are obesogenic while at the same time recognizing you need to provide alternative healthy foods that the government may need to initially subsidize? But then you can incentivize industry to develop food products that are healthy, appealing, and affordable. With human ingenuity there is no reason why we cannot do this. We have solved problems like these before. There is clear evidence that people are dying from obesity secondary to obesogenic foods. It is about time that we develop policies to counteract this.

Dr. Czernin: My final question is to you as the leader of NIDA. What are the institute's funding and research priorities for the next few years?

Dr. Volkow: Amid the current opioid crisis in America a critical goal at NIDA has been to facilitate the translation of scientific advances in our understanding of the neurobiology of addiction to expand the limited range of treatment options available for most substance use disorders (SUDs). We already have U.S. Food and Drug Administration-approved pharmacotherapies for opioid use disorder (OUD), alcoholism, and nicotine addiction, and evidencebased psychosocial treatments are available for these and other SUDs. However, the efficacy of these treatments is far from ideal. Although medications (methadone, buprenorphine, and naltrexone) significantly improve clinical outcomes and can prevent overdoses in OUD, major challenges remain regarding the use of these medications, patient compliance, and relapse rates. Thus, there is a clear need to develop extended formulations to facilitate compliance as well as new treatment strategies to enhance success with recovery. These include medications that evaluate nonopioid targets (such as dopamine D3 receptors and glutamate and cannabinoid receptors), immunotherapies (vaccines and monoclonal antibodies against opioids such as heroin and fentanyl), neuromodulation (e.g., 
transcranial magnetic stimulation and transcranial direct current stimulation), and interventions to address symptoms that trigger relapse (craving, depression, and insomnia). In parallel the NIH is prioritizing development of novel approaches to achieve analgesia for moderate-to-severe pain with low or no abuse liability.

In addition to medication development, other NIH priorities for addressing the opioid crisis are encompassed under the "Helping to End Addiction Long-term" (or HEAL) initiative, which includes services and implementation research for both OUD and pain management. This is because although many effective evidence-based interventions are available for treatment and prevention of OUD, they are not being deployed effectively. For example, only a fraction of people with OUD receive any treatment, and, of those, less than half receive the medications that are the standard of care. To fill this gap, NIDA is working with the Substance Abuse and Mental Health Services Administration to launch the HEALing Communities Study, a multisite implementation research study that will test the immediate impact of an integrated set of evidence-based interventions across health care, behavioral health, justice, and other community-based settings to prevent and treat opioid misuse and OUD within highly affected communities in 4 states.

Finally, another major initiative funded partly by HEAL, NIDA, and other NIH institutes (including the National Institute of Mental Health, National Institute on Alcohol Abuse and Alcoholism,
National Institute of Child Health and Development, National Institute of Neurologic Disorders and Stroke, National Eye Institute, National Institute of Environmental Health, National Institute on Minority Health and Health Disparities, and the Office of Behavioral and Social Science Research) is a longitudinal study to evaluate individual brain developmental trajectories in a large cohort of neonates and to follow them as they transition into adolescence while evaluating environmental exposures, including drugs and adverse social environments, behavioral and cognitive development, and genetics. This study (HEALthy Brain and Child Development) will oversample for infants exposed to opioids in utero and will serve as an open access data source so investigators can start to assess how the brain is affected by adverse social environments, mental illness, or drugs, among others. This study will complement another major longitudinal brain imaging study (Adolescent Brain Cognitive Development) supported by NIDA, NIAAA, and other NIH institutes that was launched $3 \mathrm{y}$ ago and has already enrolled nearly 12,000 youths starting at age 9 and $10 \mathrm{y}$ who will be followed until ages 20 and $21 \mathrm{y}$, with the aim to increase our understanding of how diverse experiences and exposures, including alcohol and drug use, influence adolescent brain development and behavioral outcomes.

Dr. Czernin: Dr. Volkow, thank you so much for taking the time to talk to me and our readers. 